\title{
Blaschke Sets for Bergman Spaces
}

\section{by Boris Korenblum}

Abstract.: We characterize subsets $S$ of the open unit disk $\mathbf{D}$ such that every zero sequence for a Bergman space $A^{p}, p>0$, with elements in $S$ is Blaschke.

\section{Introduction.}

The following definition is an extension of the notion of a Blaschke set introduced by Krzysztof Bogdan [B].

Definition: We call $S \subset \mathbf{D}$ a Blaschke set for a class $X$ of analytic functions on $\mathbf{D}=$ $\{z \in \mathbf{C}:|z|<1\}$ if

(i) whenever $0 \not \equiv f \in X$, and $\left\{z_{n}\right\}_{n}$ are the zeros of $f$ (counting multiplicities), with $z_{n} \in S$, the Blaschke condition holds:

$$
\sum_{n}\left(1-\left|z_{n}\right|\right)<\infty
$$

(ii) whenever $Z=\left\{z_{n}\right\}_{n}$ is a Blaschke sequence (i.e. (1) holds), with $z_{n} \in S$, there is an $f \in X$ whose zero sequence is $Z$.

REMARK: If $X$ is made up of functions of bounded Nevanlinna characteric then this definition reduces to (ii). If $H^{\infty} \subset X$, it reduces to (i).

EXAMPLES:

1. Every subset of $\mathbf{D}$ is a Blaschke set for $H^{p}, 0<p<\infty$.

2. For analytic Lipschitz classes $\operatorname{Lip}_{\alpha}(\mathbf{D}), \alpha>0$, as well as for $A^{\infty}=\left\{f: f^{(n)} \in\right.$ $\left.H^{\infty}, \forall n \geq 0\right\}$, Blaschke sets are characterized by

$$
\int_{0}^{2 \pi} \log \operatorname{dist}\left(e^{i t}, S\right) d t>-\infty
$$

where dist denotes the Euclidean distance. Note that for $\operatorname{Lip}_{\alpha}(\mathbf{D})$ and $A^{\infty}$ the zero sequences $Z$ are characterized by (1) and (2), with $S$ replaced by $Z$.

3. The Blaschke sets $S$ for the class $\mathcal{D}$ of analytic functions with finite Dirichlet integral are characterized by $(2)$ (see $[B]$ ). Note that $\mathcal{D}$-zero sequences cannot be described this way because there are $f \in \mathcal{D}$ whose zeros come arbitrarily close to every point of $\partial \mathbf{D}$ (see $[\mathrm{C}]$ and $[\mathrm{SS}]$ ).

1991 Mathematics Subject Classification, Primary 30C15 
The purpose of this paper is to obtain a description of the Blaschke sets for Bergman spaces $A^{p}(p>0)$ and growth spaces $A^{-\alpha}(\alpha>0)$. Recall that $A^{p}$ consists of functions $f$ analytic on $\mathbf{D}$ such that

$$
\|f\|_{p}^{p}=\int_{\mathbf{D}}|f(z)|^{p} \frac{d x d y}{\pi}<\infty ;
$$

$A^{-\alpha}$ consists of analytic functions $f$ with

$$
\|f\|_{-\alpha}=\sup \left\{(1-|z|)^{\alpha}|f(z)|: z \in \mathbf{D}\right\}<\infty
$$

We also consider the space $A^{-\infty}=\bigcup_{\alpha>0} A^{-\alpha}=\bigcup_{p>0} A^{p}$.

We establish the following

Theorem. A set $S \subset \mathbf{D}$ is a Blaschke set for any of the spaces $A^{p}, A^{-\alpha}, A^{-\infty}$ if and only if (2) holds.

To prove this theorem we first reduce condition (2) to a form involving a collection of disjoint "tents" tightly surrounding $S$. The sufficiency of (2) then follows from the fact that "Stolz stars" $\mathcal{S}_{F}$ are $A^{-\infty}$-Blaschke sets if the entropy $\hat{\kappa}(F)$ is finite (see (3) and [HKZ]). To prove the necessity of (2) we use some density concepts first introduced in [K1] and later refined in [S] and [HKZ].

AcKnowledgement: The suthor thanks Stefan Richter and Carl Sundberg for useful discussions. Special thanks are due to Carl Sundberg for bringing K. Bogdan's work [B] to the author's attention.

\section{An equivalent form of (2).}

We assume that $S$ contains a disk centered at 0 of radius $1 / \sqrt{2}$.

We need some terminology.

A tent is an open subset $h$ of $\mathbf{D}$ bounded by an $\operatorname{arc} I \subset \partial \mathbf{D}$ of length less than $\pi / 2$ and two straight lines through the endpoints of $I$ forming with $I$ an angle of $\pi / 4$. The closed arc $\bar{I}$ will be called the base of the tent $h=h_{I}$. A tent $h$ is said to support $S$ if $h \cap S=\phi$ but $\bar{h} \cap \bar{S} \neq \phi$. A finite or countable collection of tents $\left\{h_{n}\right\}_{n}$ is a belt if $h_{n}$

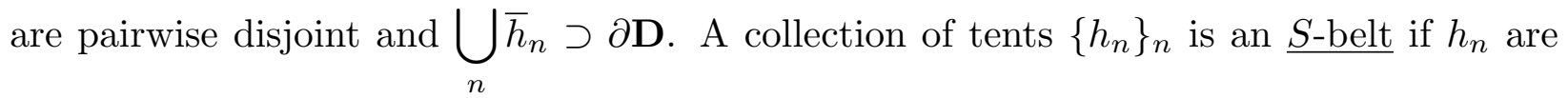
pairwise disjoint, $S$-supporting, and $\bigcup_{n} \bar{h}_{n} \supset \partial \mathbf{D} \backslash \bar{S}$. Note that an $S$-belt does not have to be a belt. If $S$ is such that $\partial \mathbf{D} \backslash \bar{S} \neq \phi, S$-belts exist: to obtain one we start at an arbitrary point $\zeta_{0} \in \partial \mathbf{D} \backslash \bar{S}$ and, moving counterclockwise, consecutively find points $\zeta_{1}, \zeta_{2} \ldots$ such that the arcs between them are the bases of $S$-supporting tents; then we proceed similarly from $\zeta_{0}$ in the opposite direction. We thus obtain a system of tents whose bases cover a 
component of $G=\partial \mathbf{D} \backslash \bar{S}$. Continuing this process for all the components we obtain an $S$-belt.

An elementary computation shows that if $h=h_{I}$ is a tent supporting $S$ then

$$
-|I| \log \frac{1}{|I|}-c|I| \leq \int_{I} \log \operatorname{dist}(\zeta, S)|d \zeta| \leq-|I| \log \frac{1}{|I|}+c|I|
$$

where $c$ is a numerical constant. We thus obtain

LEMma 1. Let $S$ be a subset of $\mathbf{D}$ such that $\partial \mathbf{D} \backslash \bar{S} \neq \phi$. Let $\left\{h_{I_{n}}\right\}_{n}$ be an $S$-belt. Then (2) holds if and only if

(A) the set $F_{0}=\bar{S} \cap \partial \mathbf{D}$ has zero Lebesgue length;

(B)

$$
\sum_{n} \kappa\left(I_{n}\right)<\infty \text { where } \kappa(I)=|I| \log \frac{2 \pi e}{|I|} .
$$

$(\kappa(I)$ is called the $\kappa$ length of $I)$.

Note that $(\mathrm{A})$ and $(\mathrm{B})$ together are equivalent to

$$
\hat{\kappa}(F)=\int_{\partial \mathbf{D}} \log \frac{2 \pi}{d(\zeta, F)}|d \zeta|<\infty
$$

where $F=F_{0} \cup \Xi$ and $\Xi$ consists of the endpoints of those bases such that $\bar{I}_{n} \subset G$; $d$ denotes the angular distance.

The quantity $\hat{\kappa}(F)$ is defined for all sets $F \subset \partial \mathbf{D}$ and is called the entropy of $F$. Closed sets with finite entropy are called Beurling-Carleson sets.

\section{Sufficiency of (3).}

Let $\Xi_{1} \supset \Xi$ consist of all endpoints of the bases $I_{n}$ (including those that are in $F_{0}$ ). Pick an increasing sequence $F_{1} \subset F_{2} \subset \ldots$ of finite subsets of $\Xi_{1}$ such that $\bigcup_{n} F_{n}=\Xi_{1}$. Then (3) implies

$$
\lim _{n \rightarrow \infty} \hat{\kappa}\left(F_{n}\right)=\hat{\kappa}(F)
$$

Each $F_{n}$ determines a belt whose tents are based on complementary arcs of $F_{n}$. Let $H_{n}$ be the union of these tents. (Note that some of these tents are not $S$-supporting because they contain ponts from $S$ ). The complement $\mathbf{D} \backslash H_{n}=\tau_{n}$ is a "Stolz Star", i.e. the union of Stolz angles with vertices in $F_{n}$ and apertures of $\pi / 2$.

Since $\hat{\kappa}\left(F_{n}\right)$ are bounded, it follows that, whenever $0 \not \equiv f \in A^{-\infty}$, the Blaschke sums for those zeros of $f$ lying in $\tau_{n}$ are bounded (see [HKZ], p. 118, Theorem 4.25). We have $\sum_{n} \tau_{n} \supset S$ and $\tau_{1} \subset \tau_{2} \subset \ldots$, which implies that the Blaschke sum for the zeros of $f$ lying in $S$ is finite. 


\section{Necessity of (3).}

Suppose now that $\hat{\kappa}(F)=\infty$. Given an arbitrary fixed $p>0$ we are going to construct a sequence $Z=\left\{z_{n}\right\}_{n}, z_{n} \in S$, such that $Z$ is an $A^{p}$-zero sequence but $\sum\left(1-\left|z_{n}\right|\right)=\infty$. In addition to the standard tools of $A^{-\infty}$-theory (density notions, premeasures, etc.) we will use some technical lemmas whose proofs are deferred to section 5 .

Recall that $F=F_{0} \cup \Xi$ where $F_{0}=\bar{S} \cap \partial \mathbf{D}$ and $\Xi$ is a finite or countable set lying in $G=\partial \mathbf{D} \backslash F_{0}$. The cluster points (if any) of $\Xi$ are in $F_{0}$.

We consider separately two cases depending on whether $\hat{\kappa}\left(F_{0}\right)$ is infinite or finite.

CASE 1: $\hat{\kappa}\left(F_{0}\right)=\infty$. By Lemma 2, s.5, there is a sequence $\left\{\zeta_{\nu}\right\}_{1}^{\infty}$ of distinct points in $F_{0}$ such that the corresponding $\operatorname{arcs}\left\{J_{\nu}\right\}_{1}^{\infty}$ between $\zeta_{\nu}$ and $\zeta_{\nu+1}$ are pairwise disjoint, cover $\partial \mathbf{D}$, i.e. $\bigcup_{\nu} \bar{J}_{\nu}=\partial \mathbf{D}$, and $\hat{\kappa}\left(\left\{\zeta_{\nu}\right\}\right)=\infty$, which is equivalent to

$$
\sum_{\nu=1}^{\infty} \kappa\left(J_{\nu}\right)=\sum_{\nu=1}^{\infty}\left|J_{\nu}\right| \log \frac{2 \pi e}{\left|J_{\nu}\right|}=\infty
$$

(Note that $\lim _{\nu \rightarrow \infty} \zeta_{\nu}=\zeta_{1}$ ). Construct a premeasure (see [K1], [K2], [HKZ]) $d \mu=p|d \zeta|-d \sigma$ whose positive part has the density

$$
p(\zeta)=\log \frac{2 \pi}{d\left(\zeta,\left\{\zeta_{\nu}, \zeta_{\nu+1}\right\}\right)}, \zeta \in J_{\nu}, \nu \geq 1
$$

and the negative singular part $-d \sigma$ puts on every point $\zeta_{\nu}$ a Dirac mass equal to $-\kappa\left(J_{\nu}\right)$. Although both positive and negative parts are infinite on $\partial \mathbf{D}, d \mu$ is $\kappa$-bounded above, which means that there is a constant $c>0$ such that for all arcs $I \subset \partial \mathbf{D}$

$$
\mu(I) \leq c|I| \log \frac{2 \pi e}{|I|} .
$$

This enables us to consider a zero-free analytic function

$$
f_{\varepsilon}(z)=\exp \left\{\varepsilon \int_{\partial \mathbf{D}} \frac{\zeta+z}{\zeta-z} d \mu(\zeta)\right\}
$$

which is in $A^{p}$ (or $A^{-\alpha}$ ) provided that $\varepsilon>0$ is sufficiently small, and $p$ (or $\alpha$ ) are arbitary but fixed positive numbers.

Now we use Lemma 3, s.5, to reduce all the singular masses at $\zeta_{\nu}$ by a factor $1 / 2$ and compensate for that by extra zeros of high multiplicity at $z_{\nu} \in S$. We can ensure that the resulting function $\Phi$ is in $A^{p}$. The zeros $z_{\nu}$ of $\phi$ (counting multiplicities) form a non-Blaschke sequence of points from $S$ (see Lemma 3 for details). 
CASE 2: $\hat{\kappa}\left(F_{0}\right)<\infty$. Then we must have $\hat{\kappa}(\Xi)=\infty$. Recall that $\Xi$ includes all the endpoint of the base arcs of the $S$-belt that are in $G=\partial \mathbf{D} \backslash \bar{S}$. Let $\left\{J_{\nu}\right\}_{\nu}$ be the sequence of these arcs arranged by decreasing lengths. Then $\hat{\kappa}\left(F_{0}\right)<\infty$ together with $\hat{\kappa}(\Xi)=\infty$ yield

$$
\sum_{\nu=1}^{\infty} \kappa\left(J_{\nu}\right)=\sum_{\nu=1}^{\infty}\left|J_{\nu}\right| \log \frac{2 \pi e}{\left|I_{\nu}\right|}=\infty
$$

It is always possible to find a decreasing sequence $1>\lambda_{1}>\lambda_{2}>\ldots \rightarrow 0$ such that

$$
\sum_{\nu=1}^{\infty} \lambda_{\nu} \kappa\left(J_{\nu}\right)=\infty
$$

Every $\bar{J}_{\nu}$ is the base of a tent $h_{J_{\nu}}$ that supports $S$; therefore there is at least one common point, say $w_{\nu}$, in $\bar{S}, \bar{h}_{J_{\nu}}$ and $\mathbf{D}$. Take every $w_{\nu}$ and repeat it $\left[\frac{\lambda_{\nu} \kappa\left(J_{\nu}\right)}{1-\left|w_{\nu}\right|}\right]$ times. Let the resulting sequence be $Z=\left\{z_{k}\right\}_{k}$.

Claim: $Z$ is a zero sequence for every $A^{p}, p>0$. To prove the claim we employ the notion

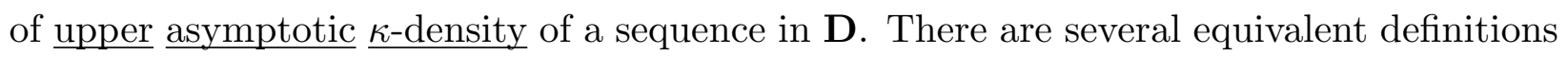
of this density. We will use the definition based on radial stars (see [HKZ]):

For an arbitrary finite set $M \subset \partial \mathbf{D}$ let $r_{M}$ denote the union of radii from 0 to points in $M$. If $A=\left\{a_{k}\right\}_{k}$ is any sequence of points in $\mathbf{D}$, we form the partial Blaschke sum for $A$ and $r_{\mathcal{M}}$ :

$$
B\left(A, r_{M}\right)=\sum_{\kappa}\left\{1-\left|a_{k}\right|: a_{k} \in r_{M}\right\}
$$

and define

$$
D^{+}(A)=\limsup _{\hat{\kappa}(M) \rightarrow \infty} \frac{B\left(A, r_{M}\right)}{\hat{\kappa}(M)}
$$

where limsup is taken over all finite $M \subset \partial \mathbf{D}$.

The following result, although short of a full characterization of $A^{p}$-zero sets, is sharp enough for our purposes.

Proposition. (See [HKZ], p.130, Theorem 4.37). Let $A=\left\{a_{\kappa}\right\}_{k}$ be a sequence of points in $\mathbf{D}$ and $\mathbf{D}^{+}(A)$ be the upper asymptotic $\kappa$-density of $A$. If $D^{+}(A)<\frac{1}{p}$ then $A$ is an $A^{p}$-zero sequence. If $D^{+}(A)>\frac{1}{p}$ then $A$ is not an $A^{p}$-zero sequence.

REMARK: This is a sharper version, due to Kristian Seip [S], of a simiilar but weaker result from $[\mathrm{K} 1]$.

Now we can prove the claim by showing that $D^{+}(Z)=0$. Let $Q=\left\{q_{\nu}=\frac{w_{\nu}}{\left|w_{\nu}\right|}\right\}_{\nu}$. Every $\operatorname{arc} J_{\nu}$ contains exactly one point from $Q$, namely $q_{\nu}$. Obviously, for computing 
$D^{+}(Z)$ we can employ only those $M$ that are finite subsets of $Q$. For such $M$ we have

$$
B\left(Z, r_{M}\right) \leq \sum_{\nu}\left\{\lambda_{\nu} \kappa\left(J_{\nu}\right): q_{\nu} \in M\right\}
$$

and

$$
\hat{\kappa}(M) \geq \sum_{\nu}\left\{\kappa\left(J_{\nu}\right): q_{\nu} \in M\right\}
$$

(see Lemma 4, s.5). Therefore

$$
\frac{B\left(Z, r_{M}\right)}{\hat{\kappa}(M)} \leq \sum_{\nu}\left\{\lambda_{\nu} \kappa\left(J_{\nu}\right): q_{\nu} \in M\right\} / \sum_{\nu}\left\{\kappa\left(J_{\nu}\right): q_{\nu} \in M\right\} .
$$

It is convenient to use the following notations:

$$
\begin{gathered}
K(M)=\sum_{\nu}\left\{\kappa\left(J_{\nu}\right): q_{\nu} \in M\right\}, \\
K_{\lambda}(M)=\sum_{\nu}\left\{\lambda_{\nu} \kappa\left(J_{\nu}\right): q_{\nu} \in M\right\} .
\end{gathered}
$$

Let $\left\{M_{n}\right\}_{n}$ be a sequence of subsets of $Q$ such that $\hat{\kappa}\left(M_{n}\right) \rightarrow \infty$. Then we have

$$
\frac{B\left(Z, r_{M_{n}}\right)}{\hat{\kappa}\left(M_{n}\right)} \leq \frac{K_{\lambda}\left(M_{n}\right)}{K\left(M_{n}\right)}
$$

Suppose that $K\left(M_{n}\right)=\mathcal{O}(1)(n \rightarrow \infty)$. Then obviously the left-hand side of (5) tends to 0 . Also, if $K\left(M_{n}\right) \rightarrow \infty$, then the right-hand (and, with it, the left-hand) side of (5) tends to 0 because $\lambda_{\nu} \downarrow 0$. Therefore every sequence $\left\{M_{n}\right\}, M_{n} \subset Q, \hat{\kappa}\left(M_{n}\right) \rightarrow \infty$, contains a subsequence $\left\{M_{n_{k}}\right\}=\left\{M_{k}^{\prime}\right\}, n_{1}<n_{2} \ldots$, such that

$$
\lim _{k \rightarrow 0} \frac{B\left(Z, r_{M_{k}^{\prime}}\right)}{\hat{\kappa}\left(M_{k}^{\prime}\right)}=0 \text {. }
$$

which implies $D^{+}(Z)=0$. Thus we have obtained a non-Blaschke $A^{p}$-zero sequence $\left\{z_{k}\right\}$ whose elements are in $\bar{S}$. Using how Lemma 5 , s.5, we can replace $z_{k}$ by nearby points $\tilde{z}_{k}$ from $S$ so that the new sequence $\left\{\tilde{z}_{k}\right\}_{k}$ is still an $A^{p}$-zero sequence and non-Blaschke. This completes the proof of the Theorem.

\section{Technical Lemma.}

We give below the statement of the technical lemmas we used in proving the Theorem, together with a brief outline of their proofs.

Definition: A sequence $\left\{\zeta_{n}\right\}_{1}^{\infty}$ of distinct points in $\partial \mathbf{D}$ is called $\underline{\mathbf{T} \text {-monotone }}$ if the open $\operatorname{arcs} I_{n}$ between $\zeta_{n}$ and $\zeta_{n+1}$ are pairwise disjoint and $\bigcup_{n} \bar{I}=\partial \mathbf{D}$. Note that it follows from this definition that $\lim _{n \rightarrow \infty} \zeta_{\infty}=\zeta_{1}$. 
Lemma 2. Every closed set $F \subset \partial \mathbf{D}$ of infinite entropy contains a $\mathbf{T}$-monotone sequence $\left\{\zeta_{n}\right\}_{n} \subset F$ of infinite entropy.

Proof: We have

$$
\hat{\kappa}(F)=\int_{\partial \mathbf{D}} \log \frac{2 \pi}{d(\zeta, F)}|d \zeta|=\infty .
$$

( $d$ denotes the angular distance). By the Heine-Borel lemma there is a point $\zeta_{0} \in F$ such that every open arc $J$ containing $\zeta_{0}$ has the property

$$
\int_{J} \log \frac{2 \pi}{d(\zeta, F)}|d \zeta|=\infty
$$

Now we can find a nested system of open arcs such that $J_{\kappa} \supset \bar{J}_{n+1}, \bigcap_{n} I_{n}=\left\{\zeta_{0}\right\}$, and a finite set $M_{k} \subset\left(J_{n} \backslash \bar{J}_{n+1}\right) \cap F$ such that

$$
\int_{J_{n} \backslash \bar{J}_{n+1}} \log \frac{2 \pi}{d\left(\zeta, M_{n}\right)}|d \zeta| \geq 1, n \geq 1 .
$$

Taking the union $E=\bigcup_{n} M_{n}$ (or a suitable subset of $E$ ) and rearranging it in a sequence will prove the Lemma.

LEMma 3. Let $f \in A^{p}(p>0)$ have an "atomic singularity" at $z=1$, i.e.

$$
\limsup _{r \rightarrow 1^{-}}(1-r) \log |f(r)|=-2 m<0 .
$$

If $m_{1}<m$ then

(i) $F(z)=e^{m_{1} \frac{1+z}{1-z}} f(z)$ is in $A^{p}$;

(ii) whenever $0 \neq \alpha_{n} \in \mathbf{D}$ and $\lim _{n \rightarrow \infty} \alpha_{n}=1$, the function

$$
f_{\alpha_{n}}(z)=\left(\frac{\alpha_{n}-z}{1-\bar{\alpha}_{n} z} \cdot \frac{\left|\alpha_{n}\right|}{\alpha_{n}}\right)^{N_{n}} F(z), \text { where } N_{n}=\left[\frac{m_{1}}{1-\left|\alpha_{n}\right|}\right],
$$

tends to $f$ in the metric of $A^{p}$.

PROOF: (i) For any $r \in(0, \infty)$ the equation

$$
\frac{1-|z|^{2}}{|1-z|^{2}}=r
$$

defines a circle $C_{r}$ internally tangent to $\partial \mathbf{D}$ at the point 1 . Such circles are called orocycles. If $f$ is in $A^{p}$ and has atomic singularity $m$ at 1 , then the function

$$
g(z)=e^{m \frac{1+z}{1-z}} f(z)
$$


may not be in $A^{p}$; however, the integral

$$
L(r)=\frac{1}{2 \pi} \int_{C_{r}}|1-\zeta|^{2}|g(\zeta)|^{p}|d \zeta|
$$

is finite and decreasing on $(0, r)$, and

$$
\int_{\mathbf{D}}|f(z)|^{p} \frac{d x d y}{\pi}=\int_{0}^{\infty} e^{-m r} L(r) d r<\infty .
$$

This implies

$$
\int_{\mathbf{D}}|F(z)|^{p} \frac{d x d y}{\pi}=\int_{0}^{\infty} e^{-\left(m-m_{1}\right) r} L(r) d r<\infty
$$

(ii) then follows by the dominated convergence theorem

LEMMA 4. If $I \subset \partial \mathbf{D}$ is an arc, $M$ is an arbitrary subset of $\partial \mathbf{D}$, and if at least one point from $M$ is in $\bar{I}$, then

$$
\int_{I} \log \frac{2 \pi}{d(\zeta, M)}|d \zeta| \geq \kappa(I)=|I| \log \frac{2 \pi e}{|I|}
$$

Proof: The minimum of the integral on the left for a given arc $I$ is attained when $M$ is a one-point set, and this point is one of the endpoints of $I$. A direct computation yields the required result.

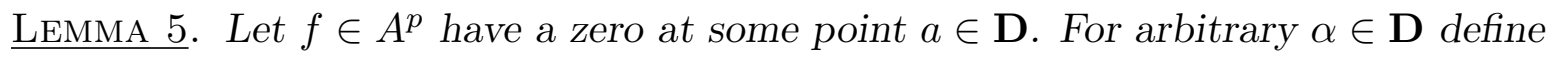

$$
f_{\alpha}(z)=\frac{B_{\alpha}(z)}{B_{a}(z)} f(z)
$$

where $B$ is a Blaschke factor:

$$
B_{\alpha}(z)=\frac{z-\alpha}{1-\bar{\alpha} z}, B_{a}(z)=\frac{z-a}{1-\bar{a} z} .
$$

Then $f_{\alpha}$ tends to $f$ in the metric of $A^{p}$ as $\alpha \rightarrow a$.

The proof is immediate and left to the reader. 


\section{$\underline{\text { References }}$}

[B] K. Bogdan. On the zeros of functions with finite Dirichlet integral. Kodai Math. J., 19 (1996), 7-16.

[C] L. Carleson. On the zeros of functions with bounded Dirichlet integral. Math. Z. 56 (1952), 289-295.

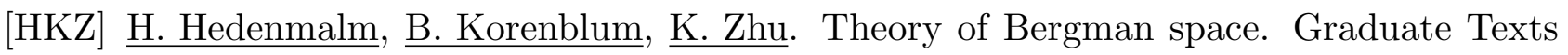
in Mathematics, 199. Springer, 2000.

[K1] B. Korenblum. An extension of the Nevanlinna theory. Acta Math. 135 (1975), 187-219.

[K2] B. Korenblum. A Beurling-type theorem. Acta Math. 138 (1977), 265-293.

[S] K. Seip. Beurling type density theorems in the unit disk. Invent. Math. 113 (1993), 21-39.

[SS] H.S. Shapiro and A.L. Shields. On the zeros of functions with finite Dirichlet integral and some related function spaces. Math. Z. 80 (1962), 217-229.

Department of Mathematics and Statistics University at Albany, State University of New York

1400 Washington Avenue, Albany, New York 12222

E-mail: borisko@math.albany.edu 